\title{
CLASSIFICATION OF ORIENTED EQUIVARIANT SPHERICAL FIBRATIONS
}

\author{
BY \\ STEFAN WANER
}

\begin{abstract}
Classifying spaces for oriented equivariant spherical fibrations are constructed, and the notion of an equivariant $S F$-fibration is introduced. It is shown that equivariant $S F$-fibrations are naturally oriented in $R O(G)$-graded equivariant singular cohomology.
\end{abstract}

Introduction. This paper is intended as a sequel to [W1], and much of the material there will be assumed here (with reference). The purpose of this note is to complete the classification theory for equivariant fibrations by constructing explicit classifying spaces for various categories of oriented equivariant spherical fibrations.

Equivariant spherical fibrations (with linear fibrewise action) are peculiar, in that two distinct fibers may look like the spheres, $S^{V}$ and $S^{W}$, of unrelated representations-even if the base space in question is connected. Thus, given a generalized $R O(G)$-graded equivariant cohomology theory $E_{G}^{*}$, there is no natural grading in which one might expect to find an orientation class. In order to "define the problem away", one notes that $E_{G}^{*}\left(S^{0}\right)$ is not concentrated in dimension zero, and proceeds according to the ideas of Wirthmuller [W7].

Let $E$ be an $R O(G)$-graded cohomology theory with products (in the sense of Wirthmuller). Let $\alpha \in R O(G)$ and let $p: X \rightarrow B$ be a $G$-spherical fibration with Thom complex $T X$. Then an element $\mu \in E_{G}^{\alpha}(T X)$ is an $E$-orientation of $p$ in dimension $\alpha$ if, for each fiber $S^{V_{x}}$ in $X$, the restriction of $\mu$ to $S^{V_{x}}$, which lies in $E_{H_{x}}^{\alpha}\left(S^{V_{x}}\right)$ with $H_{x}$ the isotropy subgroup of $p\left(S^{V_{x}}\right)$, is a generator of this free $E_{H_{x}}^{*}\left(S^{0}\right)$-module.

It may easily be seen that if $\alpha$ is an actual representation $V$, the existence of such a class implies that $E_{G}^{V}\left(S^{W}\right) \cong E_{G}^{0}\left(S^{0}\right)$ for any fiber $S^{W}$ over a stationary point in $B$. Thus the existence of an orientation implies, in general, that the cohomology theory $E$ fails to distinguish among representations of a given dimension.

On the other hand, when $B$ has connected fixed-point sets, all the fibers in $X$ must look like $S^{V}$ for some fixed $G$-module $V$ (in a sense to be made precise below). We then refer to $p$ as a $V$-dimensional fibration, and look for orientations in dimension $V \in R O(G)$. This leads to a more restricted notion of orientation, and the mathematics is correspondingly less cumbersome. There is also a natural notion of an equivariant $S F$-fibration in this context, and we show in $\$ 3$ that such fibrations

Received by the editors April 17, 1979 and, in revised form, March 17, 1981.

AMS (MOS) subject classifications (1970). Primary 54H15. 
possess natural orientations in ordinary $R O(G)$-graded equivariant cohomology with Burnside coefficients.

Although the treatment will deal with the general ( $n$-dimensional) case, we shall specialize to the $V$-dimensional case at each stage when this is of interest. In $\S 1$, generalized $\mathcal{O}_{\text {-structures }}$ [M2], of which orientations will emerge as a special case, are classified. The theory or oriented fibrations is then discussed in $\S 2$, and the main results are obtained in $\$ 3$.

I am very grateful to Professors May and McClure for their helpful suggestions, and for the many interesting discussions we had on the subject.

1. Generalized bar constructions and $\mathscr{Y}$-structures. In this section, we continue the classification theory developed in [W1] to include $\mathcal{Y}$-structures which, in turn, will be seen to include equivariant orientations. The analogous nonequivariant theory on which this will be based is due to May, [M1, §§10-11]. All the notations and definitions of [W1] will be assumed here.

Let $\left(G^{\mathcal{Q}}, F\right)$ denote an equivariant category of fibers with distinguished object set $\Lambda$ [W1, 1.1.1] and assume that we are given a fixed $G$-space $Z$, a right $\mathscr{Q}$-graph $\mathcal{Y}$ and inclusions $i_{\gamma}: Y_{\gamma} \rightarrow H_{\gamma} \mathcal{Q}\left(F_{\gamma}, Z\right)(\gamma \in \Lambda)$ such that $i=\mathrm{U}_{\gamma \in \Lambda} i_{\gamma}$ is an inclusion of right $\mathbb{Q}$-graphs. (See [W1, §2] for a discussion of graphs, the monoid $\mathbb{Q}$ associated with an equivariant category of fibers and the associated equivariant bar constructions. The notation $H$ Q d denotes the category of unbased $H$-spaces for $H<G$.)

Definition 1.1. A $\mathcal{G}_{-}$-structure $\theta$ on a $G \mathscr{F}$-space $\pi: E \rightarrow B$ [W1, 1.1] is a $G$-map $\theta$ : $E \rightarrow Z$ such that $\theta \circ \psi: F_{\gamma} \rightarrow Z$ is in $Y_{\gamma}$ for every map $\psi: F_{\gamma} \rightarrow E$ arising from a map in $P(E)_{\gamma}[\mathbf{W} 1,1.3]$ by restriction to the preimage of the identity coset of $G / H_{\gamma}$.

A $G \mathscr{F}$-map of $\mathscr{A}$-structures is a $G \mathscr{F}$-map $f: E \rightarrow E^{\prime}$, consistent with the given Q 9 -structures up to equivariant homotopy (as in $[\mathbf{M 2}, 10.1]$ ), so that $\theta^{\prime} f$ is $G$-homotopic to $\theta$ through ${ }_{S}$-structures.

$\varepsilon G \widetilde{F}\left(B, \mathscr{O}_{y}\right)$ will denote the set of equivalence classes of such structures over a given $G$-space $B$, with respect to $G \mathscr{F}$-maps of ${ }_{0}$-structures. Thus the equivalence relation is generated by the $G \mathscr{F}$-maps of $\mathcal{Y}$-structures, and not by fibrewise homotopy equivalence in general.

To deal with gammafication [W1, 1.2.3], we make the following definition.

Definition 1.2. Let $(G \mathscr{F}, F)$ be $\Gamma$-complete in the full subcategory $\mathcal{2}$ of $\mathcal{Q}$, and let $\mathscr{O}$ be a sub-right $Q$-graph of $H^{\mathcal{Q}}(\mathscr{F}, Z)=\amalg_{\gamma \in \Lambda} H_{\gamma} \mathcal{Q}\left(F_{\gamma}, Z\right)$. Then the pair $\left(\mathscr{Q}, Z\right.$ ) will be said to be admissible if $\mathcal{Q} \in \mathcal{Q}$ (that is, each $Y_{\gamma} \in \mathcal{2}$ ) and the following are valid for $G^{\mathscr{F}}$-quasifibrations $\pi: E \rightarrow B$ in 2 with $\mathscr{Y}$-structure $\theta: E \rightarrow Z$ :

(i) $\Gamma \pi: \Gamma E \rightarrow B$ admits a $\mathcal{Y}$-structure $\theta: \Gamma E \rightarrow Z$;

(ii) $\eta: E \rightarrow \Gamma E$ is a $G \mathscr{F}$-map of $\mathscr{Y}$-structures over $B$;

(iii) $\Gamma$ takes $G \mathscr{F}$-maps of $\mathscr{Y}$-structures to $G \mathscr{F}$-maps of $\mathscr{Y}$-structures.

(Equivariant quasifibrations are defined in [W2, §2], while $G \mathscr{F}$-quasifibrations are described in [W1, 1.2]. Compare the above definition with that in [M2, 10.2].)

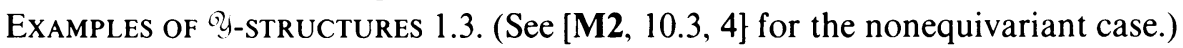

(i) Let $G^{\mathscr{F}}$ and $G^{\mathscr{Y}}$ be equivariant categories of fibers and let $j: G^{\mathscr{F}} \rightarrow G^{\mathscr{F}}$ be a functor of equivariant categories of fibers over $G$ Q . Then $j$ induces a morphism of 
monoids $\mathscr{Q} \rightarrow \mathbb{Q}^{\prime}$. Fix a $G$-action $\gamma^{\prime}$ in $G \mathscr{F}^{\prime}$, and take $\mathscr{Y}_{\gamma}$ in Definition 1.1 to be $\mathbb{Q}_{\gamma \gamma^{\prime}}^{\prime}$, so that $Z=F_{\gamma^{\prime}}$, the chosen object in $G^{\mathscr{F}^{\prime}}$. A $\mathscr{Y}$-structure on a $G \mathscr{F}$-fibration then determines a $G^{\mathscr{F}^{\prime}}$ map to $B \times F_{\gamma^{\prime}}$, where $B$ is the base space. (We are assuming that $\Lambda^{\prime} \subset \Lambda$ in order to avoid the problem of verifying that $\mathscr{Y}_{\gamma} \subset H_{\gamma} \mathcal{Q}\left(F_{\gamma}, Z\right)$ for $\gamma \in \Lambda$.) Thus a $\mathcal{Y}$-structure determines a $G \mathscr{F}^{\prime}$ trivialization of a given $G \mathscr{F}$-fibration. In particular, when $G \mathscr{F}^{\prime}$ is the subcategory of $G \mathscr{F}$ containing only those fibers of $G \mathscr{F}$ with trivial action, and $j$ is the forgetful functor, then a $\mathcal{Y}$-structure on a $G \mathscr{F}$-fibration is a trivialization of the associated nonequivariant fibration.

(ii) Let $G \mathscr{F}$ be an equivariant category of fibers, and let $f: \mathscr{B} \rightarrow \mathscr{Q}$ represent a morphism of $\Lambda$-monoids. Recall the gammafication functor which turns $G$ quasifibrations into $G$-fibrations, and let $Z=\Gamma B\left(\Lambda, \Re_{B}, \mathscr{F}\right)$, the total space of the universal $G \mathscr{F}$-fibration with reduced structure monoid $\mathscr{B}$. (Here, $\mathscr{F}$ denotes the $\Lambda$-graph determined by $G \mathscr{F}$ as in [W1]. Note that we must assume that the reduced category remains $\Gamma$-complete in the sense of [W1], as it should be for any "reasonable" choice of $\mathscr{B}$.) Now let $Y_{\gamma}=P(\Gamma B(\Lambda, \mathscr{B}, \mathscr{F}))_{\gamma}$, where $P(-)_{\gamma}$ is the principalization functor described in [W1]. Clearly $Y_{\gamma} \subset H_{\gamma} \mathcal{Q}\left(F_{\gamma}, Z\right)$, and a $\mathscr{Y}$-structure on a $G \mathscr{F}$-fibration $\pi: E \rightarrow B$ determines a commutative diagram

$$
\begin{array}{cccc}
E & \rightarrow & \Gamma B(\mathcal{L}, \Re, \mathscr{F}) \\
\pi \downarrow & & \downarrow \\
B & \rightarrow & B\left(\Lambda, \Re_{\mathcal{B}}, \mathcal{O}\right)
\end{array}
$$

and hence a reduction of the structural monoid from $\mathscr{Q}$ to $B$.

EXAMPLES OF ADMISSIBLE STRUCTURES 1.4. Consider the category $(G \mathscr{F} \mathscr{U}, F)$ of [W1, 1.1.2], with $F$ compact and $F_{\gamma} \in H_{\gamma}$ W whenever $\gamma \in \Lambda$. (Recall that $\mathcal{W}$ is the category of spaces with the homotopy type of a CW complex, while $K$ W denotes the $K$-equivariant analogue, for $K<G$.) Let $Z \in G$ UV and let $Y_{\gamma}$ be a union of any set of components of $H_{\gamma} \mathcal{Q}\left(F_{\gamma}, Z\right)$ such that $\mathcal{Y}=\amalg_{\gamma \in \Lambda} Y_{\gamma}$ is invariant under the action of $\mathcal{Q}$. Then $(\mathscr{Y}, Z)$ is an admissible pair for the category $G \mathscr{F} \mathscr{W}$. (The proof is a straightforward adaptation of the corresponding proof in [M2, 10.5], the machinery for the equivariant case having already been developed in [W1, W2] and [W3].)

REMARKS 1.5. (1) There is a based fiber variant of all of the above, the adaptation being formal and the work having essentially been done in [M2 and W1].

(2) Example 1.4 and its based variant includes the notion of orientation to be given in $\S 3$ below. (We shall tend to work with based, rather than unbased, spherical fibrations, the transition from one case to the other being purely formal.)

(3) As in [M2], we may further replace equivariant categories of fibers by equivariant categories of bundle fibers, these being described in [W1, 3.2], and obtain corresponding results for the various categories of $G$-bundles. (In this case, we replace the gamma construction by the identity transformation.)

We now give a classification theorem for $G \mathscr{F}$-fibrations with $\mathcal{Y}$-structure. For convenience, we restrict attention to the category $(G \mathscr{F} \mathscr{W}, F)$ mentioned above with the understanding that the variants corresponding to (1) and (3) above are proved by simple changes in notation. 
THEOREM 1.6 (ClASSIFICATION OF EQUIVARIANT $\mathcal{Y}$-STRUCTURES). Let $(\mathcal{Y}, Z$ ) be an

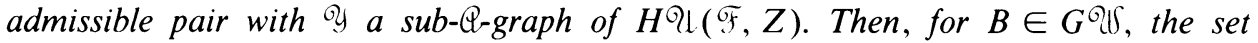
$\varepsilon G \mathscr{F}(B, \mathscr{Y})$ is naturally isomorphic to $h G \mathcal{Q}(B, B(\mathscr{Y}, \mathscr{Q}, \mathcal{O}))(=G$-homotopy classes of $G$-maps) from $B$ to $B(\mathscr{Y}, \mathcal{Q}, \mathcal{O})$, the $\mathcal{Q}$-graph $\Theta$ being as in [W1, 2.2.2(iii)]).

We give an outline of the proof, the details in the steps involved being a straightforward adaptation of the nonequivariant case in [M2, §11], as will be indicated below.

There is a natural $\mathscr{Y}$-structure on the $G \mathscr{F}$-quasifibration $p: B(\mathscr{Y}, \mathscr{Q}, \mathscr{F}) \rightarrow$ $B(\mathscr{Y}, \mathcal{Q}, \mathcal{O})$. Thus $\Gamma p: \Gamma B(\mathscr{Y}, \mathcal{Q}, \mathscr{F}) \rightarrow B(\mathscr{Y}, \mathcal{Q}, \mathcal{O})$ is a $G \mathscr{F}$-fibration with $\mathscr{Y}$-structure. This allows us to define

$$
\psi: h G \mathcal{Q}(B, B(\mathcal{Y}, \mathscr{Q}, \mathcal{O})) \rightarrow \varepsilon G \mathscr{F}(B, \mathcal{Y})
$$

by pullback. To see why it respects $G$-homotopy classes, let $h: B \times I \rightarrow B(\mathcal{Y}, \mathbb{Q}, \mathcal{O})$ be a $G$-homotopy. Then the following composite defines a $G \mathscr{F}$-homotopy of $\mathscr{Y}$ structures:

$$
f^{*} \Gamma B(\mathcal{Y}, \mathcal{Q}, \widetilde{\mathcal{F}}) \times I \rightarrow h^{*} \Gamma B(\mathcal{Y}, \mathcal{Q}, \mathscr{F}) \stackrel{\grave{h}}{\rightarrow} \Gamma B(\mathcal{Y}, \mathcal{Q}, \mathscr{F}) \rightarrow Z .
$$

(Here, the first arrow is obtained by the $G$-CHP (see [W 1, 1.2]).)

Define $\phi: \varepsilon G \widetilde{F}(B, \mathscr{Y}) \rightarrow h G \mathcal{Q}(B, B(\mathcal{Q}, \mathscr{Q},(\mathcal{O}))$ as follows. Let $\pi: E \rightarrow B$ represent an element of $\varepsilon G \mathscr{F}(B, \mathscr{Y})$, and consider the composite

$$
B \underset{g}{\stackrel{\varepsilon}{\leftrightarrows}} B(\stackrel{\oplus P}{ } E, \mathcal{Q}, \mathcal{O}) \stackrel{B \theta}{\rightarrow} B(\circlearrowleft, \mathcal{Q}, \mathcal{O})
$$

where $\varepsilon$ is a weak $G$-equivalence [W1, 2.3.1], and $g$ exists by the proof of [W1, 2.3.6], and is a right $G$-homotopy inverse of $\varepsilon$. Let $\phi([\pi])=[B \theta \circ g]$.

The proof that $\phi$ and $\psi$ are inverses is now obtained by applying the proof in [M2, 11.1] verbatim, using the results of [W1, §3] with only the following small change: In the proof of Theorem 2.3.6(d) in [W1], the map $p$ in the diagram given there with $\Lambda$ replaced by $\mathcal{O}$ remains a weak $G$-equivalence, not because of Corollary 2.3.4, but because it is a weak $G$-equivalence on each simplicial level, by the evident elaboration of Lemma 2.3.3 there.

2. Equivariant spherical fibrations and stable homotopy theory. As hinted above, equivariant orientations will emerge as $\mathcal{Y}$-structures on stable and unstable spherical $G$-fibrations. Here, we describe these fibrations with respect to certain equivariant categories of fibers, and introduce the appropriate cohomological setting for the theory of orientations.

It is technically convenient to have at our disposal a "large" ambient orthogonal $G$-space.

Definition 2.1. By $G \mathbf{R}^{\infty}$, we will mean the space $\mathbf{R}^{\infty}$ together with an ambient orthogonal $G$-action such that the following are true:

(i) $G \mathbf{R}^{\infty}$ is the limit of its finite dimensional invariant subspaces. 
(ii) Every orthogonal $G$-module (including the trivial one) occurs infinitely often (up to $G$-isomorphism).

We shall use the space $G \mathbf{R}^{\infty}$ in two ways: to select a collection of distinguished fibers for our description of equivariant spherical fibrations and to give us indexing sets for our description of $G$-spectra (see [W2, §3]).

The notation $V<G \mathbf{R}^{\infty}$ will be understood to mean that $V$ is a finite dimensional invariant subspace.

Definition 2.2. An equivariant spherical fibration of dimension $V<G \mathbf{R}^{\infty}$ is an equivariant based fibration $\pi: E \rightarrow B$ such that, for each $b \in B, \pi^{-1}(b)$ has the based $G_{b}$-homotopy type of $S^{V}$, the one-point compactification of $V$.

EXAMPLES 2.3. (i) Let $B$ be $G$-connected, meaning that, for each $H<G, B^{H}$ is connected. Then any equivariant spherical fibration in the sense of [W1] has dimension $V$ for some $V<G \mathbf{R}^{\infty}$.

(ii) More generally, let $G^{\prime}$ be some finite group which acts on a given spherical fibration $\pi$ consistently with $G$, and assume that the action of $G^{\prime}$ is transitive on the components of the fixed-point sets of $B$. Then $\pi$ is a $V$-dimensional spherical fibration for some $V$. (For example, we may take $B$ to be $S^{V}$ with tangent bundle $\pi$, and $G^{\prime}=Z_{2}$ with the natural action.)

Of course, there is a similar definition for orthogonal $G$-vector bundles of dimension $V$.

The above fibrations may be described by means of an equivariant category of fibers as follows.

Construction 2.4. Construct an equivariant category $G \mathscr{F}(V)$ of fibers as follows. The distinguished objects of $G \mathscr{F}(V)$ are the projections $p_{\gamma}: G \times{ }_{H_{\gamma}} S^{V} \rightarrow G / H_{\gamma}$, where $\left\{H_{\gamma}\right\}_{\gamma \in \Lambda}$ is a complete set of representatives of conjugacy classes of closed subgroups of $G$. We then form the associated category of based fibers as in [W1, 3.3.4]. Thus the objects of $G \widetilde{\mathcal{F}}(V)$ are $G$-maps $p: P \rightarrow Q$ with nondegenerate $G$-sections, such that $Q$ is a transitive $G$-space, and there is a fibrewise section preserving $G$-homotopy equivalence $p_{\gamma} \rightarrow p$ for some $\gamma \in \Lambda$. The morphisms of $G \mathscr{F}(V)$ are the fibrewise (section preserving) $G$-maps which restrict to a based equivariant equivalence on each fiber, with respect to the action of the appropriate subgroup on the domain.

By the results in [W1], a $G \mathscr{F}(V)$-fibration corresponds exactly to an equivariant spherical fibration of dimension $V$.

Similarly, we may define a category of equivariant bundle fibers $(G O(V) \mathscr{G}, V)$ as in [W1, 3.3.2] by taking, as our distinguished objects, the projections $p_{\gamma}: G \times_{H_{\gamma}} V \rightarrow$

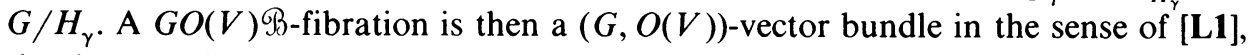
that is, an orthogonal $G$-vector bundle of dimension $V$.

Any discussion of equivariant spherical fibrations of vector bundles is incomplete without mention of possible analogues of $S F$-fibrations and $S O$-bundles. These ought to arise when the structural monoid $\mathcal{Q}$ has a reduction to an appropriate submonoid $\mathscr{B}$, as in Example 1.3(ii). It is tempting to choose $\mathscr{B}$ to be the largest submonoid for which transition to the nonequivariant case results in an $S F$-fibration. This is, however, only one of several possibilities. 
(i) SF-fibrations with G-action. Here, we construct $\mathscr{B}$ by considering only those maps $f: G \times{ }_{H} S^{V} \rightarrow G \times{ }_{K} S^{V}$ in $G \mathscr{F}(V)$ for which the map $\tilde{f}: S^{V} \rightarrow S^{V}$ given by $\tilde{f}=r f i$, where $i(v)=[e, v]$ and $r[g, w]=g w$, has degree 1 as a map of spheres. To check that this defines a submonoid, let $f: G \times{ }_{H} S^{V} \rightarrow G \times{ }_{K} S^{V}$ and $f^{\prime}: G \times{ }_{K} S^{V} \rightarrow$ $G \times{ }_{L} S^{V}$ be in $\mathcal{B}$. Write $f[e, s]=[g, \mu(s)]$ and $f^{\prime}[e, s]=\left[g^{\prime}, \mu^{\prime}(s)\right]$. Then $\overline{f^{\prime} \circ f(s)}$ $=g g^{\prime} \mu^{\prime} \mu(s)=g\left(g^{\prime} \mu^{\prime}\right) g^{-1}(g \mu(s))=g \tilde{f}^{\prime} g^{-1} \tilde{f}$, whose degree is clearly 1 .

(ii) SF-fibrations with oriented G-action. Many authors prefer, in addition, to insist that the action of $G$ itself must be orientation preserving. This amounts to a consideration of only those objects in the submonoid defined in (i) for which the action is orientation preserving. Examples include $(G, S O(n))$-vector bundles in the sense of [L1]. (An interesting criterion for such bundles has been suggested by tom Dieck; one requires that the fibrewise $n$th exterior power of a given $(G, O(n))$-vector bundle be equivalent to the trivial line bundle $B \times \mathbf{R}$, where $B$ is the base space.)

(iii) Equivariant $S F$-fibrations. As the terminology suggests, this is the variant preferred by the author. We shall say that a $G \mathscr{F}(V)$-fibration is $S F(V)$ if there is a reduction of the structural monoid which includes only those maps $f: G \times{ }_{H} S^{V} \rightarrow$ $G \times{ }_{K} S^{V}$ in $G \mathscr{F}(V)$ for which the associated map $\tilde{f}$ given in (i) is stably $H$-homotopic to the identity. This is equivalent to the requirement that $\tilde{f}^{K}$ have degree 1 for each closed subgroup $K<H$ (see [D1]), or to the requirement that $\tilde{f}$ have equivariant (stable) degree $1 \in A(H)$, the Burnside ring of $H$, as formulated by tom Dieck in [D1]. The geometry of $S F(V)$ fibrations over suitable base spaces is given as follows.

Let $p: E \rightarrow B$ be a $V$-dimensional equivariant spherical fibration. Assume that we are given an open cover of $B$ by invariant tubes of the form $G \times{ }_{H} U$, where $U \subset B$ is $H$-invariant, together with fibrewise $G$-homotopy trivializations of the form

$$
\begin{array}{ccc}
\left(G \times{ }_{H} U\right) \times S^{V} & \stackrel{\bar{\vartheta}}{\rightarrow} & E \\
\downarrow & & \downarrow p \\
G \times{ }_{H} U & \stackrel{\vartheta}{\hookrightarrow} & B
\end{array}
$$

where $G$ acts diagonally on $\left(G \times{ }_{H} U\right) \times S^{\nu}$. Let $x \in \vartheta\left(G \times{ }_{H} U\right) \cap \vartheta^{\prime}\left(G \times{ }_{H^{\prime}} U^{\prime}\right)$ for two such trivializations $\vartheta$ and $\vartheta^{\prime}$, and write $x=\vartheta[g, u]=\vartheta^{\prime}\left[g^{\prime}, u^{\prime}\right]$. Consider the following composite:

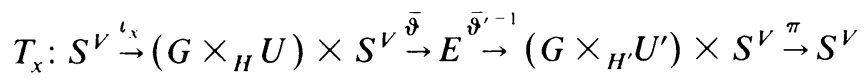

where $\iota_{x}(s)=([g, u], s), \pi([\bar{g}, \bar{u}], s)=s$ and where $\bar{\vartheta}^{\prime-1}$ is a fibrewise $G$-homotopy inverse of $\bar{\vartheta}^{\prime}$. Then $T_{x}$ is $G_{x}$-equivariant. If the system of local trivializations is such that each $T_{x}$ is stably $G_{x}$-homotopic to the identity, then $p$ is $S F(V)$-oriented by this system.

Let $p: E \rightarrow B$ be $V$-dimensional, and suppose that its fibrewise suspension by some $G$-module $W$ is $S F(V \oplus W)$. Then we shall say that $p$ is a $V$-dimensional $S F$ fibration. The theory in [W6] shows that $S F$ and $S F(V)$ coincide for large $V$, and 
that the obstruction to an $S F$ reduction over $B$ lies in Bredon cohomology, $H_{G}^{1}\left(B ; A^{*}\right)$, with coefficients in the Burnside ring units (see [W6]).

REMARKS 2.5. (i) We do not require that the action of $G$ be orientation preserving as in (ii) above.

(ii) Hauschild (private correspondence) has suggested calling a $G$-spherical fibration oriented if its restriction to each fixed-set is oriented. This is weaker than the $S F$ condition, and there are examples of non-SF $Z_{4}$-fibrations with oriented fixed-sets. The key to this discrepancy is the fact that $H_{G}^{1}\left(-; A^{*}\right)$ does not split into a product whose factors are $Z_{2}$-cohomology of the fixed-sets. This is discussed more fully in [W6].

(iii) $S F$. and $S F(V)$-fibrations provide us with (the only) examples of fibrations with orientations in ordinary ( $R O(G)$-graded) equivariant cohomology with Burnside coefficients. (This theory was developed by the author in the case of finite groups [W5], and by Lewis, May and McClure in the case of general compact Lie groups $G$ [LMMW].) The connection between $S F(V)$ orientations and equivariant singular cohomology leads one to the correct notion of equivariant orientability for $G$-manifolds. The theory of such manifolds is developed in [W6].

We now consider equivariant fibrations of nonequivariant dimension some $n$. These are $G$-fibrations in the classical sense; the preimage of a point $x$ has the $G_{x}$-homotopy type of $S^{n}$ with some orthogonal $G_{x}$ action and the covering homotopy property is satisfied equivariantly. There are also the corresponding notions of $G$-vector bundles of dimension $n$.

As in the case of $V$-dimensional fibrations, we may describe $n$-dimensional fibrations by means of an appropriate category of fibers.

Construction 2.6. Define a category $G^{\mathscr{F}_{V}}$ of fibers as follows (here, $V$ is a $G$-invariant subspace of $G \mathbf{R}^{\infty}$ of dimension $n$ ): For each closed subgroup $H<G$, choose a set of $n$-dimensional $H$-invariant subspaces of $G \mathbf{R}^{\infty} \oplus V$, including a representative of each equivalence class of $H$-modules. Let $\Lambda_{V}^{H}$ be an indexing set, and let $\Lambda_{V}=\cup_{(H) \in \psi} \Lambda_{V}^{H}$. Here, $\psi$ denotes the set of conjugacy classes of subgroups of $G$.

For each $\gamma \in \Lambda_{V}$, let $S^{V_{\gamma}}$ denote, as usual, the one-point compactification of $V_{\gamma}$. We shall take as the distinguished objects of $G \mathscr{F}_{V}$ the projections

$$
p_{\gamma}: G \times{ }_{H_{\gamma}} S^{\nu_{\gamma}} \rightarrow G / H_{\gamma}
$$

for $\gamma \in \Lambda_{V^{\gamma}}^{H^{\gamma}}$ and $V_{\gamma}$ the corresponding subspace of $G \mathbf{R}^{\infty}$. We then take $G \mathscr{F}_{V}$ to be the corresponding equivariant category of based fibers as in [W1, 3.3.4]. Thus the objects of $G \mathscr{F}_{V}$ are $G$-projections $p: P \rightarrow Q$ as in Construction 2.4, equivalent to some $p_{\gamma}$, and the morphisms are the section preserving fiber homotopy equivalences.

Similarly, we may define a category of equivariant bundle fibers $G O_{V} \Re$ as in [W1, 3.2.2] by taking our distinguished objects to be the projections $p_{\gamma}: G \times_{H_{\gamma}} V_{\gamma} \rightarrow G / H_{\gamma}$. A $G O_{V} \Re$-fibration is then an orthogonal $n$-dimensional $G$-vector bundle in the sense of [L1].

We now consider the classifying spaces for the various types of fibrations described above, and show how to pass to the stable case. (The construction of the 
classifying space associated with an equivariant category of fibers is described in [W1, 2.2.2], so that we need only recall it here.)

Firstly, in the case of $V$-dimensional spherical fibrations, we take $\mathcal{Q}(V)$ to be the $\Lambda(V)$-graph associated with the category $G \mathscr{F}(V)$. (Here, $\Lambda(V)$ is the (discrete) space $\Lambda$ of Construction 2.4.) Thus, $\mathbb{Q}(V)=\amalg_{\gamma, \gamma^{\prime}} G \mathscr{F}(V)\left(p_{\gamma}, p_{\gamma^{\prime}}\right)$. Also, let $\mathcal{O}(V)$ denote the $\mathscr{Q}(V)$-space $\amalg_{\gamma} G / H_{\gamma}$, as usual. Then the space $B(\Lambda(V), \mathscr{Q}(V), \mathcal{O}(V))$ classifies $G \mathscr{F}(V)$-fibrations. For brevity, denote this space by $B_{G} F(d)(V), B_{G} O(d)(V)$ or $B_{G} S F(d)(V)$, as the case may be.

Similarly, one may construct the classifying spaces $B\left(\Lambda_{V}, \mathscr{Q}_{V}, \hat{\theta}_{V}\right)$ in the case of $n$-dimensional spherical fibrations, and we denote these by $B_{G} F(V), B_{G} O(V)$ and so on.

Stabilization now proceeds as follows: Let $A=F, O, F(d), O(d)$ or $S F(d)$. Then define

$$
B_{G} A=\operatorname{colim} B_{G} A(V)
$$

taken over $G$-invariant $V<G \mathbf{R}^{\infty}$.

Remarks 2.7. (i) The space $B_{G} F$ differs radically from $B_{G} F(d)$ in general; the former classifies stable equivalence classes of spherical $G$-fibrations $\left(\pi\right.$ and $\pi^{\prime}$ are stably equivalent if they become equivalent after addition of (possibly different) trivial fibrations to each), while the latter classifies stable classes of spherical $G$-fibrations with dimension some $V<G \mathbf{R}^{\infty}$.

(ii) The space $B_{G} O(d)$ gives us a new version of equivariant $K$-theory:

Let $\tilde{K}_{G}(d)(X)=\left[X^{+}, B_{G} O(d)\right]_{G}$ ("reduced equivariant $K$-theory with dimension"); $K_{G}(d)(X)=\left[X^{+}, B_{G} O(d) \times R O(G)\right]_{G}$ ("unreduced equivariant $K$ theory with dimension").

The latter is readily seen to coincide with the Grothendieck group of virtual classes of $G$-vector bundles with dimension $V$ for some $V<G \mathbf{R}^{\infty}$. By Example 2.3(i), it follows that $K_{G}(d)$ and $K_{G}$ coincide on $G$-connected spaces, so that, in particular, $K_{G}(d)(*) \cong K_{G}(*) \cong R O(G)$.

(iii) The inclusion of classifying spaces induces a monomorphism $\sigma: K_{G}(d)(X) \rightarrow$ $K_{G}(X)$ for every finite $G$-CW complex $X$, and the cokernel is classified by the universal space corresponding to a reduction of the structural monoid as in Example 1.3(ii).

To conclude this section, we describe the cohomological setting in which equivariant orientations take place.

Definition 2.8. An equivariant $R O(G)$-graded spectrum is a collection of based $G$-spaces $E(V)$, indexed on $V<G \mathbf{R}^{\infty}$, together with (based) structure $G$-maps

$$
\sigma: \Sigma^{W} E(V) \rightarrow E(V+W) \text { for } V \perp W,
$$

whose adjoints, $\tilde{\sigma}: E(V) \rightarrow \Omega^{W} E(V+W)$, are $G$-homeomorphisms. We also require that the following diagrams commute for each triple $W, V, Z$ of mutually orthogonal f.d. sub- $G$-modules of $G \mathbf{R}^{\infty}$ : 


$$
\begin{array}{ccc}
\Sigma^{Z} \Sigma^{W} E(v) & \stackrel{\Sigma^{Z} \sigma}{\rightarrow} & \Sigma^{Z} E(V+W) \\
\downarrow \cong & & \downarrow \sigma \\
\Sigma^{Z+{ }^{W}} E(V) & \stackrel{\sigma}{\rightarrow} & E(Z+V+W) \\
\Sigma^{Z} \Sigma^{W} E(V) & \rightarrow & E(Z+W+V) \\
\downarrow T & & \downarrow \\
\Sigma^{W} \Sigma^{Z} E(V) & \rightarrow & E(W+Z+V)
\end{array}
$$

where $T$ denotes the twist map.

A $G$-spectrum $E$ is said to be an equivariant ring spectrum (in the sense of Lewis and May) if we are also given structure $G$-maps $\phi: E(V) \wedge E(W) \rightarrow E(V+W)$ for $V \perp W$, and $\varepsilon: S^{V} \rightarrow E(V)$ which define a $G$-homotopy associative and unital structure. (If one prefers, one may pass to the stable category of Lewis and May, define the smash product, $E \wedge E$, and then define a spectrum as a pairing $E \wedge E \rightarrow E$ in the stable category.)

Each equivariant ring spectrum determines a multiplicative $R O(G)$-graded cohomology theory $E_{G}^{*}(-)$ by defining, for each based $G$-complex $X$,

$$
E_{G}^{V-W}(X)=\left[\Sigma^{w} X, E(V)\right]_{K} \text {, with unit } 1=[e] \in E_{G}^{0}\left(S^{0}\right) .
$$

Further, one may formally pass to an $R O(H)$-graded $H$-equivariant ring spectrum, and hence cohomology theory, by defining

$$
E(V)=\operatorname{colim} \Omega^{V^{\prime}} E(W), \quad \text { over } G \text {-invariant } W<G \mathbf{R}^{\infty},
$$

where $V^{\prime}$ denotes the orthogonal complement of $V$ in $W$.

REMARKS 2.9. The following facts are immediate consequences of the definitions:

(i) $E_{G}^{*}\left(G \times{ }_{H} X / G \times_{H^{*}}\right)=E_{H}^{*}(X)$ for any based $H$-complex $X$;

(ii) $E_{G}^{*}\left(S^{V}\right)$ is a free $E_{G}^{*}\left(S^{0}\right)$-module on a single generator, by the suspension isomorphism, for any $V<G \mathbf{R}^{\infty}$.

3. Equivariant orientations. Let $E$ be an equivariant commutative ring spectrum, let $G \mathscr{F}$ be one of the categories of fibers described in $\S 2$, let $\alpha \in R O(G)$ and let $\pi$ be a $G \mathscr{F}$-fibration.

Definition 3.1. An $E$-orientation of $\pi$ in dimension $\alpha$ is a class $\mu \in E_{G}^{\alpha}(T Y)$ (where $\pi: Y \rightarrow B$ ) which restricts to a generator of the free $E_{G_{x}}^{*}\left(S^{0}\right)$-module $E_{G_{x}}^{*}(T X)$ for each fiber $X=\pi^{-1}(x)$ of $\pi$. An orientation preserving map $f: Y \rightarrow Y^{\prime}$ of fibrations is required to preserve this structure up to homotopy. (Compare the definition in [M1].)

We now show that orientations are special cases of $\mathscr{Y}$-structures (cf. [M2, III.2] for the nonequivariant case).

Proposition 3.2. Every E-orientation of $\pi$ in dimension $\alpha$ determines a $\mathcal{Y}$-structure on $\pi$ in a natural way such that equivalences of $\mathcal{Y}$-structures coincide with equivalences of E-orientations in dimension $\alpha$. 
Proof. Let $\Lambda$ denote the indexing space for $G^{\mathscr{F}}$, as in $\S 2$, and take $\mathscr{Y} \subset$ $\amalg_{\gamma \in \Lambda}\left(\Omega^{V_{\gamma}} E(\alpha)\right)^{H_{\gamma}}$ to be the union of all components corresponding to generators of $E_{H_{\gamma}}^{*}\left(S^{V_{\gamma}}\right)$ as an $E_{H_{\gamma}}^{*}\left(S^{0}\right)$-module (for each $\gamma \in \Lambda$ ). To check that this is an $\mathbb{Q}$-algebra, one verifies that composition with fibrewise $G$-homotopy equivalences sends generators to generators. Let $Z=E(\alpha)$. Then $(\mathcal{Y}, Z)$ is an admissible pair so that, by Theorem 1.6, $E$-oriented equivariant spherical fibrations are classified by $B(\mathcal{Y}, \mathbb{Q}, \mathcal{Q})$.

REMARKS 3.3. (i) In order to handle $V$-dimensional $S F$-fibrations, we may, in the case of orientations in dimension $V \in R O(G)$, be more restrictive. Let $S \mathscr{Y}(V)$ be the disjoint union (over $\gamma \in \Lambda$ ) of the identity components of the spaces $\left(\Omega^{V} E(V)\right)^{H_{\gamma}} \cong$ $E(0)^{H_{r}}$. We may then consider $S F(V)$-fibrations with $\mathcal{Y}(V)$-structures. These correspond to the classical notion of orientability in the nonequivariant case when $E$ arises from ordinary cohomology. The classifying space for these orientations is $B(S \mathcal{Y}(V), S \mathscr{Q}(V), \mathcal{O}(V))$, where $S \mathscr{Q}(V)$ is the (reduced) monoid for $S F(V)$-fibrations and where $\mathcal{O}(V)$ is given in Construction 2.6. To check that $S \mathcal{Y}(V)$ is an $S \mathscr{Q}(V)$ graph, let $r \in\left(\Omega^{V} E(V)\right)^{H}$ be in the identity component of $E(0)^{H}$ and let $f$ : $G \times{ }_{K} S^{V} \rightarrow G \times{ }_{H} S^{V}$ be in $S \mathscr{Q}(V)$. Write $f[e, s]=[g, \mu(s)]$. Then $f(r)(s)=\operatorname{gr\mu }(s)$ $=\left(\mathrm{grg}^{-1}\right)(g \mu)(s)$, where $\mathrm{grg}^{-1} \in E(0)^{K}$ is in the identity component, and where $s \mapsto g \mu(s)$ is stably $K$-homotopic to the identity, by definition of $S \mathscr{Q}(V)$.

(ii) In general, the existence of an orientation in the sense of Definition 3.1 for an equivariant spherical fibration $\pi$ implies strong restrictions on either $\pi$ or the spectrum $E$; if, for example, $\alpha=V$ and $S^{W}$ occurs as a fiber in $\pi$, then we must have $E_{G}^{V}\left(S^{V}\right) \cong E_{G}^{V}\left(S^{W}\right)$. Thus the fibers of $\pi$ must be indistinguishable under the theory $E$. However, when $\mathscr{F}=\mathscr{F}(V)$, this problem vanishes, and we naturally seek $E$ orientations in dimension $V$.

By analogy with May [M1], we have the canonical projection

$$
q: B(\mathcal{Y}, \mathbb{Q}, \theta) \rightarrow B(\Lambda, \mathbb{Q}, \mathcal{\theta})
$$

which forgets the orientation.

The map $q$ is not an equivariant quasifibration, although the homotopy type of the fixed-point sets of its homotopy-theoretic fiber may be determined as follows.

Consider the map $q^{H}: B(\mathcal{Y}, \mathcal{Q}, \mathcal{O})^{H} \rightarrow B(\Lambda, \mathcal{Q}, \mathcal{Q})^{H}$ in the case of $n$-dimensional spherical fibrations with $E$-orientations. One may compare it with a quasifibration as follows.

Let $R_{H}$ be a complete set of representatives of equivalence classes of linear actions of $H$ on $S^{n}$, and denote the representative corresponding to $\gamma$ by $S^{V_{r}}$.

Proposition 3.4. There are weak equivalences $f, \bar{f}$ making the following diagram commute:

$$
\begin{array}{ccc}
\operatorname{II}_{\gamma \in R_{H}} B\left(Y_{\gamma}, A_{\gamma}, *\right) & \stackrel{\bar{f}}{\rightarrow} & B(\mathcal{Y}, \mathcal{Q}, \mathcal{\theta})^{H} \\
\downarrow \mathrm{II}_{\gamma} q_{\gamma} & & \downarrow q^{H} \\
\operatorname{II}_{\gamma \in R_{H}} B\left(*, A_{\gamma}, *\right) & \stackrel{f}{\rightarrow} & B(\Lambda, \mathcal{Q}, \mathcal{\theta})^{H}
\end{array}
$$

Here, $A_{\gamma}$ is the monoid of $H$-equivariant homotopy equivalences on $S^{V_{\gamma}}, Y_{\gamma} \subset$ $\left(\Omega^{V_{\gamma}} E(\alpha)\right)^{H}$ consists of those maps giving rise to a generator as usual, and $q_{\gamma}$ is the natural quasifibration with quasifiber $Y_{\gamma}$. 
Proof. The maps $f$ and $\bar{f}$ are induced by choosing $R_{H}$ as a subset of $\Lambda$, and then taking the natural inclusion

$$
y_{\gamma}\left[g_{n}, \ldots, g_{1}\right]^{*} \mapsto y_{\gamma}\left[g_{n} \times_{H} 1, \ldots, g_{1} \times_{H} 1\right] e H,
$$

where $g_{i} \times{ }_{H}$ 1: $G \times{ }_{H} S^{V_{\gamma}} \rightarrow G \times{ }_{H} S^{V_{\gamma}}$, and $e H$ denotes the identity coset in $G / H$. That $f$ and $\bar{f}$ are weak equivalences follows from the fact that the spaces on the left classify fibrations and oriented fibrations $H$-equivariantly over base spaces with trivial $H$-action.

When we restrict to $V$-dimensional fibrations, the situation is much simpler, the left-hand spaces reducing to $q: B(Y(V), A(V), *) \rightarrow B(*, A(V), *)$, where $A(V)$ is the monoid of $H$-equivalences $S^{V} \rightarrow S^{V}$, and $Y(V) \subset\left(\Omega^{V} E(V)\right)^{H}$ corresponds to units of $E_{H}^{0}\left(S^{0}\right)$.

If we further restrict to $S F(V)$-fibrations in the sense of Remark 3.3(i), we may use Proposition 3.4 to deduce the existence of canonical orientations for $S F$ - and $S F(V)$-fibrations in ordinary cohomology, as alluded to in Remark 2.5(iii).

COROLLARY 3.5. Let $E$ be the spectrum for ordinary equivariant cohomology with Burnside coefficients (thus $\left(\Omega^{V} E(V)\right)^{H} \sim A(H)$, the Burnside ring of $\left.H\right)$, let $\alpha=V$ and let $G \mathscr{F}$ be the category of fibers for $S F(V)$-fibrations. Then, with $\mathcal{Y}=S \mathcal{Y}(V)$ (as in Remark 3.5(i)), and $\theta=O(V)$, the map $q$ is a weak G-equivalence.

Proof. By Proposition 3.4, it suffices to show that each $Y_{\gamma}$ is contractible. But here, $Y_{\gamma}$ is the component of $\left(\Omega^{V} E(V)\right)^{H_{\gamma}}$ corresponding to the unit in $E_{H_{\gamma}}^{0}\left(S^{0}\right)=$ $A\left(H_{\gamma}\right)$, and is contractible since we are using ordinary cohomology.

COROLlary 3.6. Every $S F(V)$-fibration has a V-dimensional orientation in ordinary equivariant cohomology with Burnside coefficients.

Of course, analogous results exist for $S O(V)$ - and $S U(V)$-bundles, the proofs in these cases being a notational variation of those above.

Finally, we consider passage to the stable case. In the case of $V$-dimensional fibrations, denote the $V$-dimensional classifying space for $E$-oriented fibrations by $B_{G}(F(V), E)$. The structure of the spectrum $E$ then allows us to pass to the limit, colim $B_{G}(F(V), E)=B_{G}(F, E)$, and similarly for $O(V), S F(V), S O(V)$ and so on. In the case of fibrations without equivariant dimension, the notion of a stable class of orientations in dimension $\alpha$ is meaningless in general, although in the special case of cohomology theories which completely ignore differences among representations, it is possible to formulate an ad hoc version of $B_{G}(F, E)$.

In [W6], the theory of $G$-orientations is applied to smooth " $V$-dimensional manifolds", or manifolds whose tangent bundles admit $V$-dimensional structures. Orientation and Thom classes are also constructed there, and the relationship between the geometry of special $V$-structures and equivariant algebraic functors is explored.

\section{BIBLIOGRAPHY}

[D1] T. tom Dieck, Transformation groups and representation theory, Lecture Notes in Math., vol. 766, Springer-Verlag, Berlin and New York, 1979.

[LMMW] G. Lewis, J. P. May, J. McClure and S. Waner, Ordinary equivariant cohomology theory, Univ. of Chicago (in preparation). 
[M1] J. P. May, $E_{\infty}$ ring spaces and $E_{\infty}$ ring spectra, Lecture Notes in Math., vol. 577, Springer-Verlag, Berlin and New York, 1977.

[M2] Classifying spaces and fibrations, Mem. Amer. Math. Soc. No. 155 (1975).

[M3] __ Homotopic foundations of algebraic topology, Mimeographed notes, Univ. of Chicago.

[W1] S. Waner, Equivariant classifying spaces and fibrations, Trans. Amer. Math. Soc. 258 (1980), 385-405.

[W2] , Equivariant fibrations and transfer, Trans. Amer. Math. Soc. 258 (1980), 369-383.

[W3] , Equivariant homotopy theory and Milnor's theorem, Trans. Amer. Math. Soc. 258 (1980), $351-368$

[W5] _ _ RO(G)-graded equivariant singular cohomologv, preprint, Univ. of Virginia, 1980.

[W6] _ _ Oriented G-manifolds, preprint, Univ. of Virginia, 1980.

[W7] R. Wirthmuller, Equivariant homology and duality, Manuscripta Math. 11 (1974), 373-390.

[MHW] J. P. May, H. Hauschild and S. Waner, Equivariant infinite loop space theory (in preparation).

[L1] R. Lashof and M. Rothenberg, G-smoothing theory, Proc. Sympos. Pure Math., vol. 32, part 1, Amer. Math. Soc., Providence, R. I., 1978, pp. 211-266.

Department of Mathematics, University of Virginia, Charlottesville, Virginia 22903 